\title{
M odel os assistenciais na saúde suplementar: o caso de uma operadora de autogestão
}

\author{
Care models in the supplementary care system: \\ the case of a self-management operator in Brazil
}

Deborah Carvalho $\mathrm{M}$ alta ${ }^{1}$

Alzira deOliveiraJorge ${ }^{2}$

${ }^{1}$ Departamento M aterno Infantil eSaúde Pública, Escola deEnfermagem, UFM G. Av. Alfredo Balena, 190/ 50 andar, Santa Efigênia. 30130-100 Belo H orizonte M G. dcmalta@uol.com.br ${ }^{2}$ Secretaria M unicipal de Saúde de Belo H orizonte.
Abstract This paper analyzes the care model employed by a self-management operator in Brazil. The investigation was carried out in form of a case study. Different actors, managers of the $O$ perator and the Call Center and affiliated hospitals and physicians, were interviewed for collecting data about the care model provided. The in depth analysis of the interviews used the following analytical dimension for evaluating the collected data: objective of the actors, instituted policies, instituted technological knowledge, organizational form and offered care. Differently from themarket logic, the investigated self-management operator showed not profit-driven in itsoperations but seeks market strategies such as capturing new clients, adopting regulatory measures and controlling introduction of new technologies to ensure his survival. The operator has encouraged health promotion and client bonding. These results refer exclusively to the analyzed operator and do not apply to the existing operators in general.

Key words Health insurance, Governmental regulation, Care models
Resumo 0 trabalho analisa o modelo assistencial deuma operadora de planos desaúde deautogestão. Adotou-sea metodologia de estudos decasos, sendo realizadas entrevistas, com diferentes atores: dirigentes da operadora e do call center, prestadores hospitalares e médicos, visando coletar informaç̧ões sobre o modelo assistencial praticado. Procedeu-se à análise das entrevistas e foram adotadas as seguintes dimensões na avaliação das informações coletadas: objetivo dos atores, política instituída, saberes tecnológi cos instituídos, forma organizativa eassistência prestada. Dentre os resultados, a operadora investigada apresentou objetivos distintos da lógica do mercado, não visando o lucro nas operações. A mesma tem buscado estratégias de mercado para a sobrevivência, como por exemplo, captação de novos clientes, adoção de medidas regulatórias, controle na introdução de novas tecnologias. A operadora tem incentivado a adoção de prática de promoção da saúde e vinculação de clientela, especialmente junto à públicos específicos. Por tratar-se de estudo de caso, os resultados referem-se à operadora analisada, não contemplando a totalidadedasoperadoras de planos.

Palavras-chave Planos e seguros saúde, Regulação pública, M odelos assistenciais 
Introdução

A atuação do Estado na saúde suplementar tem como marco a aprovação da Lei $n^{\circ}$ 9.656/98, que estabeleceu um novo patamar no processo de regulação ${ }^{1}$. Entretanto, há que se aprofundar a natureza dessa regulação, visando garantir a qualidade da assistência à saúde e a produção do cuidado integral neste setor. Coloca-se o desafio de entender como se estruturam os modelos assistenciais vigentes na saúde suplementar, identificando o modo como as operadoras de planos de saúde vêm se organizando para a oferta dos serviços de saúde, de forma a garantir a assistência com qualidade aos seus usuários, responsabilizando-se pelo seu processo saúde/doença.

Existe uma grande heterogeneidade nos padrões de qualidade, prestação de serviços e modelos assistenciais praticados, bem como na forma de organização do setor. Entretanto, o tema de modelos assistenciais na saúde suplementar tem sido pouco investigado no Brasil. 0 atual trabalho busca avançar nesta compreensão, analisando a forma de organização da assistência em uma operadora de autogestão.

A atenção à saúde suplementar no Brasil é composta pelos segmentos das autogestões, medicinas de grupo, seguradoras e cooperativas. Denomina-se "autogestão" os planos próprios patrocinados ou não pelas empresas empregadoras, constituindo o segmento não comercial do mercado de planos e seguros. As autogestões totalizam cerca de 310 empresas e aproximadamente 5,4 milhões de beneficiários, ou cerca de 12,2\%. 0 grupo éheterogêneo, incluindo as grandes indústrias de transformação, entidades sindicais, empresas públicas e até empresas com pequeno número de associados. Cerca de $50 \%$ são administrados por instituições sindicais ou entidades jurídicas paralelas às empresas empregadoras, como as caixas de assistência, caixas de previdência e entidades fechadas de previdência. Integram sua administração representantes dos trabal hadores e patronais. Percentual significativo é administrado por departamentos de benefícios/recursos humanos da própria empresa ${ }^{2-6}$.

O segmento comercial da saúde suplementar compreende as cooperativas de trabal ho médico - Unimeds e cooperativas odontológicas, as empresas de medicina de grupo e as seguradoras. As seguradoras vinculadas ou não a bancos representam a modalidade empresarial mais recente no mercado de assistência médica suple mentar e detêm cerca de $10,4 \%$ do contingente de pessoas cobertas através de planos privados de saúde. As cooperativas de trabalho médico, as Unimeds, possuem cerca de $26,8 \%$ dos clientes de planos de saúde e se organizaram a partir da iniciativa de médicos, com a argumentação da ameaça de perda da autonomia da prática médica e da mercantilização da medicina ${ }^{7}$. As medicinas de grupo, constituídas inicialmente por grupos médicos aliados ao empresariado paulista, são atualmente responsáveis por quase $36,2 \%$ dos ben eficiários da assistência médica supletiva. Esse segmento se organizou em torno de proprietários/acionistas de hospitais, criando redes de serviços e credenciando hospitais e laboratórios, dado que existia um comprador de serviços que Ihes garantia um mercado seguro. 0 surgimento do setor deu-se a partir de meados da década de 1960, com o denominado convênio-empresa entre a empresa empregadora e a empresa médica (medicina degrupo), estimulados pela Previdência Social, que repassava subsídios per capita pelo serviço prestado, prática essa que foi decisiva no empresariamento da medicina. N este segmento, incluem-se também as odontologias de grupo ${ }^{4}$.

\section{M etodologia}

Como proposta metodológica utilizou-se o estudo de caso que, segundo $\mathrm{Hartz}^{8}$, são indicados quando se deseja examinar o conjunto das relações existentes entre diferentes variáveis necessárias à compreensão de um fenômeno complexo, em situações em que o investigador tem pouco controle sobreos acontecimentos ou quando setrabal ha com uma problemática contemporânea inserida no contexto social.

Contandriopoulos et al. ${ }^{9}$, citado por $\mathrm{Hartz}^{8}$, afirmam que a potência explicativa destes estudos não decorre da quantidade de observações, mas da profundidade eda análise do caso. Quanto à validade interna, os estudos de caso são avaliados pela qualidade de articulação teórica na qual se apóia a pesquisa e pela adequação entre os modos de análise utilizados e modelo teórico escolhido. Com relação à validação externa, eles não têm a pretensão de alcançar uma generalização estatística, mas o interesse de general izar um quadro teórico ou modelo relacionado à compreensão de um determinado problema em diferentes situações ${ }^{8}$.

Quanto à coleta de dados, foram realizadas entrevistas com diferentes atores: dirigentes da operadora e do call center, prestadores hospitalares e médicos, visando coletar informações sobreo modelo assistencial praticado. Procedeu-se 
ainda à análise de base de dados existentes na ANS através do Sistema de Informação de Beneficiários e Cadastro de Operadoras 5,6 .

Procedeu-seao contato com a operadora, que indicou os dirigentes que participaram da entrevistas, ao todo três dirigentes. Além disso, foram selecionados os diretores dos dois maiores prestadores de serviços hospitalares, aferidos junto à operadora, segundo a maior produção de serviços realizados. Os mesmos foram escolhidos dentre as linhas de cuidado assistencial investigadas (assistência materno infantil e cardiologia). Foram ainda selecionados dois médicos destas especialidades, relacionados no catálogo de prestadores da operadora e que prestavam serviços na região próxima aos hospitais selecionados. Foram entrevistados ainda outros dois médicos que trabalhavam no serviço próprio da operadora. Ao todo foram realizadas nove entrevistas, sendo cinco com prestadores médicos (um clínico, dois cardiologistas, um pediatra e um ginecologista), segundo as linhas de cuidado assistencial investigadas, três dirigentes da operadora e um coordenador do call center.

As entrevistas foram conduzidas buscando apreender o model o assistencial emecanismos de regulação praticados. Utilizou-se, para isso, questionários semi-estruturados. As entrevistas buscaram aproximar-se do objeto pesquisado e abordaram temas como o modelo assistencial praticado, a oferta de serviços, a existência de serviços de prevenção/promoção, a inclusão de novas tecnologias eformas de avaliação de efetividade deagregação de novas tecnologias, as fontes deinformação utilizadas, o acesso dos usuários à rede de serviços, as formas de avaliação da satisfação do usuário e os mecanismos de regulação utilizados. Os pesquisadores visitaram ainda o call center da operadora, observando os fluxos e demandas.

A pesquisa foi submetida ao Consel ho deÉtica em Pesquisa (COEP) da Universidade Federal de $M$ inas Gerais (UFM G), os instrumentos utilizados nas entrevistas foram submetidos previamente à pré teste e todas as entrevistas foram conduzidas pelos próprios pesquisadores, procedendo-se ao esclarecimento dos objetivos da mesma, apresentando os questionários a serem utilizados eo termo delivre consentimento, sendo queesteúltimo foi lido, esclarecido e assinado por cada um dos entrevistados.

Para a análise das entrevistas, foram realizadas repetidas leituras das mesmas e agrupadas as falas de acordo com a finalidade dos elementos constitutivos. As entrevistas com dirigentes das operadoras e prestadores possibilitaram definir marcadores e parâmetros de avaliação. Foram utilizados conceitos teóricos para relacionar as categorias empíricas com as analíticas, buscando fundamentação na bibliografia existente.

$\mathrm{Na}$ análise do conteúdo das entrevistas, adotou-se o conceito de M erhy, Cecílio e N oguei ra ${ }^{10}$ sobre modelos assistenciais, que define: modelo técnico assistencial constitui-se na organização da produção de serviços a partir de um determinado arranjo de saberes da área, bem como de projetos de ações sociais específicos, como estratégias políticas de determinado agrupamento social. Entendemos desse modo, que os model os tecno-assistenciais estão sempre apoiados em uma dimensão assistencial e tecnológica, para expressar-se como projeto de política, articulado a determinadas forçase disputas sociais ${ }^{10}$.

Foram adotadas as seguintes dimensões analíticas para avaliação das informações coletadas: objetivo dos atores, política instituída, saberes tecnológicos instituídos, forma organizativa eassistência prestada.

Os objetivos dos atores foram trabalhados segundo a definição dos propósitos que instituem o segmento, ou seja, a missão institucional.

A dimensão política foi caracterizada pelos atores implicados em cada segmento eseus interesses disputantes. Buscou-se identificar as relações do ator (que dirigea operadora), a sua forma deoperar junto aos demais atores em cena, a relação com aANS (ressarcimento ao SUS, regulação, segmentação), a relação com o SUS, a relação com os prestadores e usuários (informações, participação eavaliação), parcerias eas disputas no mercado como a incorporação de novas tecnologias.

Os saberes tecnológicos foram apontados dentre os pressupostos que sustentam e direcionam a organização.

A dimensão organizativa buscou caracterizar a forma de organizar a rede assistencial, os mecanismos de regulação utilizados junto aos prestadores e beneficiários, o sistema de informação utilizado, dentre outros.

A dimensão assistencial avaliou as práticas assistenciais estabelecidas pela operadora, a existência de ações de promoção e prevenção à saúde, a integralidade da atenção à saúde, a existência de projetos de desenvolvimento técnico-científico ou de capacitação, a adoção de práticas de gerenciamento do cuidado, a assistência farmacêutica, internação domiciliar, alta complexidade, assistência à saúde mental, saúde do trabaIhador e outros. 
Resultados ediscussão

A análise do material empírico coletado procurou elementos nas falas dos entrevistados que, apoiados no marco teórico relatado, possibilitaram a compreensão do modelo assistencial praticado pela operadora selecionada. A poiados na matriz analítica descrita anteriormente, apresenta-se, a seguir, os principais resultados das entrevistas.

Os objetivos da operadora de autogestão investigada são distintos da lógica do mercado, por não visar o lucro nas operações, isto é, as receitas são utilizadas no custeio da própria atividade. A empresa mantenedora contribui para o financiamento do plano, além da contribuição dos seus beneficiários, o que implica, como afirmado por Bahia ${ }^{3}$, em assumir o risco da variabilidade dos gastos com o consumo de serviços de saúde, ou seja, reter o risco. A ênfase, portanto, não é a atividade lucrativa, mas proporcionar assistência à saúde de seus funcionários e dependentes. Ainda segunda a mesma autora, a década de 1990 para as autogestões caracterizou-se pelo aumento da participação dos empregados no custeio dos seus planos, pela restrição dos critérios de el egibilidade de dependentes e pela utilização de co-pagamento para o consumo dos denominados pequenos riscos, visando redução de consumos desnecessário de serviços. Pesquisa Nacional CIEFAS 2000 identificou que o custeio dos planos nas autogestões é realizado predominantemente através de processo de co-participação entreempresas eempregados, em cerca de $80,7 \%$ das empresas pesquisadas, confirmando a tendência apontada?2.

$\mathrm{Na}$ análise da dimensão política, com relação às disputas e estratégias de mercado, tendo em vista a crise financeira que abala as operadoras, ficou evidenciado que a autogestão estudada tem buscado o desenvolvimento de novas estratégias para sobrevivência dentro do mercado de saúde suplementar. Para isso, foi introduzido, na década de 1990, uma nova modalidade de planos, visando a expansão da carteira entre os familiares dos associados e ex-associados, que conquistou novos beneficiários, cobrindo parte dos custos do plano de associados. O utra estratégia que tem se expandido constitui-se no produto ofertado pela operadora para a prestação de assistência médica e/ou hospitalar a empregados de empresas privadas, órgãos públicos, universidades, fundações e/ou outras entidades afins (saúde empresa). Este produto ainda é pequeno em termos de adesões, mas existe definição da operadora pela sua ampliação.
Os dirigentes da operadora discutem a necessidade de um novo formato de incorporação de novas tecnologias, dado que custos crescentes das mesmas têm afetado muito o mercado; sendo assim, a operadora defende que a ANS regule o processo de incorporação de novas tecnologias ao rol de procedimentos da ANS, bem como os reajustes necessários à esta incorporação. Outro ponto defendido pela mesma consiste na ampliação do escopo do marco regulatório da ANS para atingir a regulação assistencial de prestadores (hospitalares, clínicas e laboratórios), avaliando sua qualidade buscando maior controle e garantia de qualidade na prestação dos serviços.

$\mathrm{N}$ as entrevistas, foram apresentadas sugestões ao órgão regulador (ANS), como a criação de uma câmara técnica específica para as autogestões onde fosse possível a discussão de ferramentas para o gerenciamento do cuidado a partir deexperiências concretas eintrodução denovo formato de acompanhamento das operadoras através de indicadores de saúde, visando avaliar as melhorias produzidas na assistência à saúde.

Esta operadora avalia positivamente a regulação pública produzida no setor, o que teria resultado em avanço para o mercado. Entretanto, relata o impacto administrativo produzido, resultando também em aumento nos custos operacionais. 0 ressarcimento ao SUS, que consiste em mecanismo adotado pela ANS, por lei, que obriga as operadoras a devolverem ao SUS OS recursos financeiros utilizados nos atendimentos realizados por seus clientes na rede SUS, é defendido pela operadora ${ }^{11}$. Afirma que a relação com o SU Socorre pontualmentena articulação de parcerias, como por exemplo, do combate à dengue.

$\mathrm{N}$ as entrevistas com médicos e prestadores hospitalares, quando indagados da sua avaliação da operadora, em geral, a mesma foi bem avaliada, quando comparada com outras.

Os usuários exercem o controle social sobre os serviços prestados, participando regionalmente através dos conselhos dos usuários eleitos e das Conferências de Saúde dos Conselhos a cada dois anos, na qual são definidas as principais diretrizes a serem adotadas. Os dirigentes desta autogestão avaliam que a participação social tem sido uma experiência satisfatória, onde os beneficiários dentre outras coisas participam com agentes de multiplicação da informação de uma forma rápida, funcionando como se fosse um "marketing de rede". Os Consel hos são vistos como parceiros e ocupam um papel importante 
na definição de prioridades de investimento e também no enfrentamento e disputas junto ao mercado. Os contatos da operadora com os beneficiários se dão através de: serviço de atendimento tel efônico, boletim informativo, site, mala direta, jornal, conselhos deliberativo efiscal, além dos consel hos de usuários.

A operadora realiza parcerias com diferentes organizações e empresas, tanto na questão ambiental, quanto em temas referentes à saude, tais como campanhas educativas como a dengue, estimulando adesões voluntárias de seus beneficiários. Foram realizadas ainda parcerias com instituições de caráter científico, órgãos formadores, universidades e M inistério da Saúde para a discussão de protocolos clínicos, campanhas de promoção à saúde, dentre outras.

Os saberes tecnológicos utilizados estão centrados em diversos campos, como os da Clínica e da Epidemiologia Clínica, especialmentea medicina baseada em evidências, lançando-se mão também da Economia em Saúde e Administração. Destaca-se o campo da Saúde Coletiva, com os conhecimentos do Planejamento e Administração em Saúde.

Quanto à dimensão organizativa, a operadora trabalha com um mix de rede própria e conveniada no atendimento aos seus beneficiários. Entretanto, o grande peso dos seus gastos e prestadores de serviços concentra-se na sua extensa rede conveniada, em todo o país. A rede própria é organizada no modelo de unidade básica desaúde, utilizando clínicos, pediatras, gine cologistas, cirurgião geral, assistente social, e outros. Recentemente, introduziu-se a experiência das equipes de saúde da família. Esta nova proposta assistencial objetiva as ações de promoção, focando na atenção primária e na coordenação do cuidado dos usuários a partir dos seus serviços próprios.

A extensa rede de prestadores dificulta a capacidade de regulação da operadora e reduz a possi bilidade da garantia da qualidade dos serviços prestados. No novo modelo assistencial proposto, busca-se reorganizar a rede prestadora a partir dos serviços próprios, utilizando-a como porta de entrada para reordenar o fluxo. Buscase um referenciamento mais racional e uma assistência à saúde mais integral e cuidadora. Esta prática já está em curso em al gumas áreas como na saúde mental, tratamento da obesidade, diabetes, e lerdort (lesão por esforços repetitivos).

Os mecanismos de regulação utilizados pela operadora para a gestão dos prestadores são: as auditorias médicas realizadas junto aos presta- dores hospitalares, os boletins técnicos informativos normatizando a incorporação tecnológica, as centrais de aten dimento para autorizações dos procedimentos e as tabel as de serviços. Entretanto, a ferramenta regulatória mais potente encontra-se em implantação e consiste na articulação dos serviços próprios como porta de entrada com uma rede de referência designada. A rede própria deverá referenciar e regular a rede contratada.

O sistema de informações foi reestruturado, mas ainda predomina a análise de custos e o processamento de pagamentos.

Os médicos revelam que não existem regras em relação à produtividade, seja no controle de número de consultas definidas por turno de trabalho ou solicitação de exames. Avalia-se que a operadora não se pauta pela redução do custo em detrimento da qualidade, apesar de avaliarem o custo da consulta médica baixo, com na maioria das operadoras. Alguns médicos do quadro próprio relatam não uniformização de condutas na auditoria prévia, resultando em diferentes decisões para casos semelhantes. Das entrevistas realizadas, observa-se que o controle sobre o trabalho médico é pequeno, existindo grande liberdade na solicitação e autorização de exames, procedimentos e internações.

Os protocolos clínicos, via de regra, não são adotados, à exceção de al guns casos priorizados pela autogestão como nas linhas de cuidado da oncologia, idosos e lerdort. No caso da oncologia, define-setipo, frequência efluxo de procedimentos, além do encaminhamento à rede referenciada. $\mathrm{Na}$ lerdort, da mesma forma, são seguidas normas definidoras dos fluxos de atendimentos, rede referenciada. 0 restante dos protocolos existentes não são exigência da operado$\mathrm{ra}$, mas iniciativa dos prestadores hospitalares ou médicos apoiados nas escolas médicas ou sociedades de especialidades.

$\mathrm{Na}$ dimensão assistencial, a operadora estudada encontra-se em processo inicial de mudança do modelo de assistência através da implantação de equipes de saúde da família em seus serviços próprios. Os referenciais adotados são os do modelo de promoção e têm sido implantados mecanismos visando o aumento do vínculo, a responsabilização e acolhimento ao usuário. As práticas de promoção e prevenção ofertadas são: vinculação e adscrição de clientela à equipes de programa de saúde da família da rede própria, equipes multidisciplinares que desenvolvem atividades de promoção e melhoria da qualidade de vida dos usuários através de grupos operativos, práticas alternativas como acupuntura, ho- 
meopatia, atividades educativas e de promoção à saúde veiculadas através de jornal, site, boletins e grupos operativos.

O gerenciamento do cuidado é praticado nas linhas de cuidado consideradas estratégicas. Cada uma dessas linhas tem conhecimentos específicos e conta com gestores do cuidado para realizar este trabal ho de monitoramento, articulação e integração da assistência. Algumas linhas de cuidado são trabalhadas dentro da rede própria, sendo queem outras se definiu uma rede de prestadores referenciada e seidentificou para essa clientela um fluxo preferencial.

Em função da clientela fechada equetem progressivamente envelhecido, com alta incidência de doenças crônico-degenerativas, os serviços próprios da operadora adquiriram competência técnica em áreas como a assistência ao idoso, trabal hando o estímulo à qualidade de vida, desenvolvendo assistência integral incluindo a criação de centro de convivência para idosos e outros programas educativos e/ou assistenciais. Para os portadores de lerdort (lesão por esforços repetitivos), criou-se uma porta de entrada no serviço próprio, que consiste no cadastramento do usuário e a partir daí o planejamento do cuidado, enviando-o para os determinados serviços, articulando a assistência e a qualidade do atendimento prestado nos diferentes níveis de atenção.

A saúde mental foi reorganizada visando articular os recursos necessários à atenção específi$\mathrm{ca}$, incluindo 0 acompanhamento e oferecimento de políticas substitutivas ao manicômio após a desospitalização.

São desenvolvidos projetos de internação domiciliar, junto a pacientes idosos, portadores de doenças crônicas, pacientes neurológicos e oncológicos e ainda aqueles submetidos a processo de desospitalização na área de saúde mental. Esses pacientes são acompanhados por mé dicos referenciados, identificados junto à rede prestadora dereferência. Isso foi confirmado por meio de entrevistas com médicos que participam das equipes de atenção domiciliar da operadora.

$\mathrm{Na}$ oncologia, motivados pelos altos custose má qualidade da assistência desen volvi da anteriormente, foi instituído um novo fluxo assistencial, visando a articulação do cuidado e sua regulação. Esse trabalho resultou na definição de uma rede articulada de prestadores, que se inicia com a implantação da notificação dos casos oncológicos, definição de protocolos de condutas e fluxos para a oncologia. Essa estratégia tem melhorado a qualidade da assistência prestada junto à rede de referência da operadora. A operadora oferta coberturas mais amplas que aquela prevista no rol de procedimentos da saúde suplementar como, por exemplo, oferecendo o transplante de medula óssea.

Os médicos entrevistados confirmaram a adoção de acompanhamento de determinadas patologias estimuladas pela operadora.

Quanto à assistência farmacêutica, observouse que são ofertados medicamentos específicos e deuso contínuo quesão $70 \%$ financiados pela operadorae $30 \%$, pelo usuário. Osremédios paraAIDS e câncer são totalmente pagos pela operadora.

\section{Conclusão}

Este trabal ho constitui parte de pesquisa que investigou os modelos de assistência à saúde suplementar utilizados por operadoras selecionadas, de 2003 a 2004.

A utilização da metodologia deestudo decaso trouxe a vantagem de um aprofundamento para melhor conhecimento do objeto a ser investigado, mas, pelo seu caráter, não tem a pretensão de ser generalizável e sim de identificar um quadro teórico ou modelo para compreensão do objeto ede suas características essenciais ${ }^{8,12}$. Assim, esta pesquisa traça o perfil de uma operadora de plano de autogestão, mas certamente não contempla a totalidade das operadoras existentes e a multiplicidade de realidades, cujos resultados devem ser analisados com o cuidado e a singularidade exigidos.

A operadora aqui analisada tem apresentado experiência positiva no que tangeà vinculação de clientela, definição de cuidadores, aliada a outras estratégias degrande visibilidade, como por exemplo, a da saúde da família. $\mathrm{Na}$ operacional ização da vinculação de seus usuários, a operadora buscou instituir entre seus quadros alguns profissionais que fazem a gestão do cuidado de grupos vulneráveis (idosos, oncologia, saúde mental e lerdort), organizando 0 atendimento através de uma rede melhor referenciada e tendo a rotina de sempre acompanhar o "caminhar" desse usuário por essa rede credenciada. São agendados retornos nos quais se avaliam novas programações, novos fluxos, buscando a integralidade, continuidade e o acompanhamento do caso.

I dentifica-se que esta operadora disponibiliza coberturas bem mais amplas que as outras do mercado, ofertando serviços além do previsto na Lei $n^{\circ} 9.656$ que regulamenta o setor ${ }^{1}$. Em al guns casos, como o da saúde mental, constitui uma das poucas operadoras que disponibiliza dispo- 
sitivos substitutivos à prática manicomial. Isso confirma as características já apontadas para as autogestões da sua missão não ter como ênfase a atividade lucrativa, mas proporcionar assistência à saúde de seus funcionários e dependentes.

A vinculação do usuário vem norteando 0 planejamento da operadora, visando constituirse em estratégia fundante e estruturante da assistência, o que será buscado com a disseminação do programa de saúde da família.

A estratégia de saúde da família tem também o objetivo de remodelar e rearticular toda a assistência, inclusive visando à redução de custose definição de uma rede prioritária de prestadores. Entretanto, a realidade atual do modelo de atenção egastos implementados ainda se pautam pela fragmentação e pelo esgotamento desse modelo consumidor de procedimentos, com custos elevados e resultados nem sempre adequados. $\mathrm{N}$ esse sentido, procura-se investir em práticas mais cuidadoras e de promoção à saúde do usuário.
Esta opção feita para obter melhores resultados deverá ser aprofundada pelo aumento da capacidade de investimento de recursos financeiros, ampliando a equipe de acompanhamento qualificada, que possi bilite a construção de vínculose investimento na promoção à saúde.

Torna-se necessário avaliar a efetividade das mudanças em curso, seja no ordenamento do fluxo e regulação dos prestadores, avaliando-se a redução de custos e o resultado assistencial produzido.

Por fim, observou-se que a operadora pesquisada tem desenvolvido diversas iniciativas importantes para a produção de uma assistência mais integral e cuidadora, além de um investimento no gerenciamento e monitoramento de pacientes de risco. Há que se acompanhar este movimento buscando avaliar e monitorar os resultados, podendo a médio prazo se constituir em referência para o setor, difundindo estas boas práticas para outras operadoras.

\section{Colaboradores}

DC M alta e AO Jorge participaram igualmente de todas as etapas da elaboração do artigo.

\section{Agradecimentos}

Gostaríamos de agradecer ao financiamento e apoio institucional da Agência N acional deSaúde Suplementar (ANS) eàAlineAzevedo Bianchetti, Alessandra Pinheiro Caminhas eAdriano $\mathrm{M}$ arçal Pimenta, que participaram da transcrição de fitas e organização da base de dados. 


\section{Referências}

1. Lei no 9.656 de 3 de junho de 1998. Dispõe sobre os Planos de Assistência à Saúde. Diário O ficial da U nião 1998; 4 jun.

2. Comitê de Integração de Entidades Fechadas (CIEFAS). Pesquisa Nacional sobre Saúde nas Empresas. São Paulo: CIEFAS; 2000.

3. Bahia L. Planos privados de saúde: luzes e sombras no debate setorial dos anos 90. Cienc Saude Colet 2001; 6(2):329-339.

4. Malta DC, Cecílio LCO, M erhy EE, Jorge AO, Franco $T$, Costa MA. Perspectivas da regulação na saúde suplementar diante dos modelos assistenciais. Cienc Saude Colet 2004; 9(2):433-444.

5. Agência Nacional de Saúde Suplementar. Cadastro de O peradoras. Rio de Janeiro: ANS; 2006.

6. Agência Nacional de Saúde Suplementar. Sistema de Informação de Beneficiários - SIB. Rio de Janeiro: ANS; 2006.

7. Duarte CMR. UNIMED: história e características da cooperativa de trabalho médico no Brasil. Cad Saúde Pública 2001; 17(4):35-41.

8. Hartz ZM A, organizador. Avaliação em Saúde: dos modelos conceituais à prática na análise da implantação de programas. Rio de Janeiro: Fiocruz; 1997.
9. Contandriopoulos AP, Champagne F, Potvin BL, Denis JL, Boyle P. Savoir préparer une recherche. La définir, la structurer, la financer. Montréal: Les Presses de I'U niversité de M ontréal; 1990.

10. Merhy EE, Cecílio LCO, Nogueira RC. Por um modelo tecno-assistencial da política de saúde em defesa da vida: contribuição para as Conferências de Saúde. In: Cadernos da $9^{\circ}$ Conferência Nacional de Saúde; 1992 out. 10-13; Brasília: GTTCO; 1992. p.30.

11. Brasil. M inistério da Saúde. M anual de ressarcimento ao SUS. Brasília: M inistério da Saúde; 2000. [M imeo]

12. Gonçalves RBM. Tecnologia e organização social das práticas de saúde. São Paulo: Hucitec; 1994.

Artigo apresentado em 22/05/2006

Aprovado em 18/06/2007

Versão final apresentada em 30/07/2007 